\title{
Can we all just get along?
}

\begin{abstract}
Familiarity with developments in all areas of genetics-from the trained intuition of dysmorphology to algorithms for SNP calling in next-generation sequencing-is extraordinarily productive in research and clinical translation. Actively organized conferences modeled on the recent European Society of Human Genetics (ESHG) meeting in Nuremberg go a long way in preparing the field for future success.
\end{abstract}

saw a clinician's insight save the cost of a whole-genome sequence in a matter of seconds. Four hundred clinical geneticists (including a few fascinated spectators, such as myself) had crowded into a lecture theater for an hour in unknown territory. A clinical genetics workshop has a simple formula: each presenter gets two slides and two minutes to present an unsolved case. Each presentation is focused on a genetic condition under investigation for which there is incomplete information: no positive genotype test, insufficient evidence of transmission, an atypical presentation or, indeed, sometimes a condition that is completely unknown to medicine.

During the presentation, a second screen projects reference images from POSSUM (http://www.possum.net.au/) and the Winter-Baraitser Dysmorphology Database from the London Medical Databases (http://www.lmdatabases.com/), and it is often one of these images that triggers an insight from one of the experts. Two factors make these watchable events. First, it is an audience of several hundred clinical geneticists, any one of whom might provide the solution. "What happens when you throw the kid in the air?" "He giggles." With this exchange, a key syndromic feature is identified. Absence of the vestibular apparatus of the inner ear is the reason this child can get such a lot of fun out of a playground swing without getting dizzy! Second, the consultation is chaired by Dian Donnai, whose panache and timing have elevated this forum to an educational event-part courtroom drama, part unexpected family reunion-that mystery writers cannot equal. Comments are delivered in shorthand, fruitless hypotheses pruned and redirected. Often, someone in the room has worked with another family with a related condition. Nowadays, it is likely there is someone who has sequenced exomes or genomes from a similar family and can offer a candidate gene to test for mutations. There is a feeling of excitement in the room as collaborations are formed before our eyes to bring genomic technology to previously unknown syndromes.

Just as experience with rare disorders can guide the genomic search for mutations, genomic tools from genome-wide association studies (GWAS) and genome sequencing are changing our understanding of the way in which mutations contribute to Mendelian disorders. Structural variants (in particular, small deletions) are still relatively difficult to identify by array genotyping or sequencing, but it is particularly interesting to see the regions most prone to de novo rearrangement in disease in their evolutionary context. The most dynamic and dangerous regions of the genome may also be the most productive for the evolution of our species, if I have understood correctly the hypothesis of this year's Mendel Prize lecturer, Evan Eichler (for example, Nat. Genet. 39, 1361-1368, 2007). On pages 872 and 881 of this issue, the evolutionary history of the large 17q21 inversion and its associated structural rearrangements is further described.

Deleterious or clinically interesting variants are now regularly discovered, both in population samples and incidentally in the exomes of individuals ascertained for a different disease, for example, in the ClinSeq project (Am. J. Hum. Genet. published online 14 June 2012; doi:10.1016/j.ajhg.2012.05.021). We predict that these population findings will increasingly challenge pedigree-based estimates of the penetrance of disease-linked variants and that the currently illdefined concept of 'pathogenicity of a gene variant' will give way to a less ambiguous interpretation grounded in statistical support similar to that used in fine mapping rare and common variants in association studies. Even within a Mendelian disorder, different aspects of the phenotype can respond to different genetic modifiers. Some are common enough to be identified by GWAS. The rarer ones can be found by the strategy of exome sequencing from the extremes of a population. There is a nice example of this strategy on page 886 of this issue, in which a modifier of the frequency and severity of bacterial lung infection was found in individuals with cystic fibrosis.

We commend the organizers of this year's ESHG meeting for anticipating the integrated future of genomic medicine and for organizing their meeting so as to showcase the usefulness of each strand of genetics research to the others. This is a vision of the future of the discipline that is close to the one we promote in this journal. 